\title{
Upper airway gene expression reveals a more robust innate and adaptive immune response to SARS-CoV-2 in children compared with older adults
}

\section{Eran Mick}

University of California, San Francisco https://orcid.org/0000-0002-7299-808X

\section{Alexandra Tsitsiklis}

University of California, San Francisco https://orcid.org/0000-0003-3467-1171

Natasha Spottiswoode

University of California, San Francisco

\section{Saharai Caldera}

Chan Zuckerberg Biohub

\section{Paula Serpa}

University of California, San Francisco

\section{Angela Detweiler}

Chan Zuckerberg Biohub

\section{Norma Neff}

Chan Zuckerberg Biohub https://orcid.org/0000-0001-7141-5420

\section{Angela Oliveira Pisco}

Chan Zuckerberg Biohub https://orcid.org/0000-0003-0142-2355

\section{Lucy Li}

CZ Biohub

\section{Hanna Retallack}

University of California, San Francisco

\section{Kalani Ratnasiri}

Chan Zuckerberg Biohub https://orcid.org/0000-0001-5953-0004

\section{Kayla Williamson}

University of Colorado

\section{Victoria Soesanto}

University of Colorado

\section{Eric Simões}

University of Colorado School of Medicine, Aurora, CO, USA

\section{A. Kistler}

Chan Zuckerberg Biohub

\section{Brandie Wagner}


University of Colorado

Joseph DeRisi

CZ Biohub

\section{Lilliam Ambroggio}

University of Colorado

Peter Mourani

Arkansas Children's

Charles Langelier ( $\sim$ Chaz.Langelier@ucsf.edu )

University of California, San Francisco https://orcid.org/0000-0002-6708-4646

\section{Article}

Keywords: COVID-19, SARS-CoV-2, coronavirus, age, children, adults, transcriptomics, nasopharyngeal

Posted Date: August 26th, 2021

DOl: https://doi.org/10.21203/rs.3.rs-784784/v1

License: (c) (i) This work is licensed under a Creative Commons Attribution 4.0 International License. Read Full License

Version of Record: A version of this preprint was published at Nature Communications on July 8th, 2022. See the published version at https://doi.org/10.1038/s41467-022-31600-0. 
1 Upper airway gene expression reveals a more robust innate and adaptive

2 immune response to SARS-CoV-2 in children compared with older adults

4 Authors

5 Eran Mick $^{1,2,3,{ }^{*},}$ Alexandra Tsitsiklis ${ }^{1,{ }^{*}}$, Natasha Spottiswoode ${ }^{1}$, Saharai Caldera ${ }^{1,3}$, 6 Paula Hayakawa Serpa ${ }^{1,3}$, Angela M. Detweiler ${ }^{3}$, Norma Neff ${ }^{3}$, Angela Oliveira Pisco ${ }^{3}$, 7 Lucy M. $\mathrm{Li}^{3}$, Hanna Retallack ${ }^{4}$, Kalani Ratnasiri ${ }^{3}$, Kayla M. Williamson ${ }^{5}$, Victoria Soesanto ${ }^{5}$, 8 Eric A. F. Simões ${ }^{6}$, Amy Kistler $^{3}$, Brandie D. Wagner ${ }^{5,6}$, Joseph L. DeRisi ${ }^{3,4}$, Lilliam Ambroggio ${ }^{6}$, 9 Peter M. Mourani ${ }^{6, \dagger, \neq}$, Charles R. Langelier ${ }^{1,3, \neq,+}$

\section{Affiliations}

121 Division of Infectious Diseases, University of California, San Francisco, CA, USA

132 Division of Pulmonary and Critical Care Medicine, University of California, San Francisco, CA, 14 USA

153 Chan Zuckerberg Biohub, San Francisco, CA, USA

164 Department of Biochemistry and Biophysics, University of California, San Francisco, CA, USA

175 Department of Biostatistics and Informatics, Colorado School of Public Health, University of 18 Colorado, Aurora, CO, USA

6 Department of Pediatrics, Children's Hospital Colorado and University of Colorado, Aurora, CO, USA

† Present address: Arkansas Children's Research Institute, Arkansas Children's Hospital, Little Rock, AR, USA

$24 *$ These authors contributed equally

$25 \neq$ These authors jointly supervised this work

$26+$ Correspondence to: chaz.langelier@ucsf.edu

27

28 Keywords

29 COVID-19, SARS-CoV-2, coronavirus, age, children, adults, transcriptomics, nasopharyngeal 


\section{Abstract}

Unlike other respiratory viruses, SARS-CoV-2 disproportionately causes severe disease

32 in older adults and only rarely in children. To investigate whether differences in the upper airway

33 immune response could contribute to this disparity, we compared nasopharyngeal gene

34 expression in 83 children (<19-years-old; 38 with SARS-CoV-2, 11 with other respiratory viruses,

3534 with no virus) and 154 adults (>40-years-old; 45 with SARS-CoV-2, 28 with other respiratory

36 viruses, 81 with no virus). Expression of interferon-stimulated genes (ISGs) was robustly activated

37 in both children and adults with SARS-CoV-2 compared to the respective non-viral groups, with

38 only relatively subtle distinctions. Children, however, demonstrated markedly greater upregulation

39 of pathways related to $\mathrm{B}$ cell and $\mathrm{T}$ cell activation and proinflammatory cytokine signaling,

40 including TNF, IFNY, IL-2 and IL-4 production. Cell type deconvolution confirmed greater

41 recruitment of $B$ cells, and to a lesser degree macrophages, to the upper airway of children. Only

42 children exhibited a decrease in proportions of ciliated cells, the primary target of SARS-CoV-2,

43 upon infection with the virus. These findings demonstrate that children elicit a more robust innate

44 and adaptive immune response to SARS-CoV-2 infection in the upper airway that likely

45 contributes to their protection from severe disease in the lower airway. 


\section{Introduction}

One of the defining features of the COVID-19 pandemic has been the striking relationship between disease severity and age $\mathrm{e}^{1-3}$. While infection with other respiratory viruses, such as

49 influenza or respiratory syncytial virus (RSV), causes significant morbidity and mortality in both 50 young children and older adults ${ }^{3-9}$, severe COVID-19 occurs disproportionately in older adults 51 and only very rarely in children ${ }^{1,3,10-14}$. A recent comprehensive modeling study estimated that the 52 infection fatality rate is lowest for children ages 5-9 $(\sim 0.001 \%)$ and that even adults in their 40 s 53 may already be at 100-fold greater risk of death from COVID-191. The age-dependent effect on 54 disease severity and mortality has been shown even when accounting for age-associated 55 comorbidities $^{15}$.

A few studies have examined differences in systemic immunological profiles of hospitalized children and adults with COVID-19, revealing greater breadth and neutralizing activity of SARS-CoV-2 specific antibodies as well as stronger CD4+ T cell responses to viral spike protein in adults ${ }^{16,17}$. These studies suggested that poorer outcomes in adults are not due to failure to engage a systemic adaptive immune response, and that children may exhibit a less pronounced

61 adaptive response because their infection course is milder. One study reported higher serum 62 levels of IL-17A and interferon-y (IFNy) in children early in their hospitalization ${ }^{17}$, suggesting a 63 more robust innate immune response may contribute to the milder disease course.

Our understanding of age-related differences in the immune response at the site of initial 65 infection, the upper airway, remains limited. Several large-scale studies have found no systematic 66 differences between children and adults in the distribution of SARS-CoV-2 viral load measured in 67 nasopharyngeal (NP) swabs ${ }^{18,19}$, suggesting children are not generally better able to control viral 68 replication in the upper airway. However, differences in the upper airway microenvironment and 69 immune response could still contribute to protection from severe disease in children, for example, 70 by limiting migration of the virus into the lower airway. 
Small-scale studies have begun to compare the upper airway immune response to SARS-

72 CoV-2 in children and adults, with some contradictory results. One study found that children with

73 COVID-19 expressed higher levels of genes associated with innate immune pathways, including

74 interferon-stimulated genes and genes related to NLRP3 inflammasome signaling ${ }^{20}$. Another

75 study, however, found no age-related differences in interferon-stimulated gene expression and

76 reported globally similar host transcriptional responses between adults and children with diverse

77 types of viral infections ${ }^{21}$, highlighting the need for further investigation. Importantly, neither study

78 directly controlled for SARS-CoV-2 viral load when comparing immune-related gene expression

79 between children and adults.

We previously used metagenomic RNA-sequencing of NP swabs to compare upper airway

81 gene expression in adult patients with COVID-19, other viral acute respiratory illnesses or non-

82 viral illnesses ${ }^{22}$. Our analysis revealed a pronounced interferon response in COVID-19 patients,

83 proportional to SARS-CoV-2 viral load, but attenuated activation of additional innate immune and

84 pro-inflammatory pathways compared to patients with other viral infections ${ }^{22}$. Here, we report new

85 sequencing data from a relatively large pediatric cohort to enable assessment of age-related

86 differences in the upper airway transcriptional response to SARS-CoV-2 infection and shed further

87 light on these outstanding questions. When controlling for viral load, our results suggest that

88 differences in the overall magnitude of interferon-stimulated gene expression in the upper airway

89 of children and adults with COVID-19 are relatively subtle and seem unlikely to explain their

90 distinct clinical outcomes. However, we also find clear evidence of more robust pro-inflammatory

91 and adaptive immune responses in the upper airway of children, which may contribute to their

92 protection from severe disease.

93

\section{Results and Discussion}

95 To compare the upper airway gene expression response to SARS-CoV-2 infection in 96 children and adults, we utilized our previously published dataset of NP swab RNA-sequencing 
97 from an adult cohort alongside newly sequenced swabs from a pediatric cohort. All samples in

98 both cohorts were obtained in the course of clinical testing for SARS-CoV-2 by reverse 99 transcription polymerase chain reaction (RT-PCR) at the University of California San Francisco 100 or Children's Hospital Colorado. We included patients up to 19 years of age in the pediatric cohort 101 and restricted the adult cohort to those 40 years of age or older to impose clearer age separation.

102 As in our previous analysis, we divided each age cohort into three viral status groups:

103 1) patients with PCR-confirmed SARS-CoV-2 infection (“SARS-CoV-2" group), 2) patients 104 negative for SARS-CoV-2 by PCR with no other pathogenic respiratory virus detected by 105 metagenomic RNA sequencing (“No Virus” group), and 3) patients negative for SARS-CoV-2 who 106 had another respiratory virus detected by sequencing (“Other Virus” group). Finally, we limited 107 the samples in the SARS-CoV-2 group to those with at least 10 viral reads-per-million (rpM), 108 comparable to PCR $C_{t}$ values below $30^{22}$, which have been associated with recovery of actively 109 replicating virus from respiratory specimens ${ }^{23-25}$.

110 The final dataset included 83 children (38 SARS-CoV-2, 34 No Virus, 11 Other Virus; 111 median age 4, IQR 2-12) and 154 adults (45 SARS-CoV-2, 81 No Virus, 28 Other Virus; median 112 age 62, IQR 47-71) (Figure 1A,B; Supplemental Table 1A-C). There were no statistically 113 significant differences between children and adults with respect to the site of clinical testing 114 (Supplemental Table 1A). Most of the patients in the SARS-CoV-2 group in both age cohorts 115 were tested as outpatients, indicative of an early/mild stage of disease (Supplemental Table 1A). 116 Samples in the SARS-CoV-2 group in both age cohorts spanned several orders of magnitude of 117 viral load, and while viral load trended higher in the children this did not reach statistical 118 significance (Figure 1C). Rhinovirus was the most prevalent other respiratory virus detected in 119 both age cohorts and a diversity of other viruses were also identified (Figure 1D).

120 We began by performing differential expression (DE) analyses between the SARS-CoV-2 121 and No Virus groups within each age cohort separately. This approach minimizes confounding by 122 age-related gene expression differences unrelated to SARS-CoV-2 infection and any potential 
123 batch effects, though it could be affected by differences among the patients in each cohort's No

124 Virus group. The analyses yielded 1,961 and 1,216 differentially expressed genes at an adjusted $125 \mathrm{p}$-value $<0.1$ for the pediatric and adult cohorts, respectively (Supplemental Data 1). We then 126 performed gene set enrichment analyses ${ }^{26}$ (GSEA) using Gene Ontology (GO) biological process 127 annotations ${ }^{27}$ on the DE results in each cohort and compared the enriched pathways.

128 As expected, a range of immune related pathways were upregulated in both adults and 129 children with SARS-CoV-2 compared to those with no virus (Figure 2A; Supplemental Data 2).

130 Pathways related to the interferon response appeared as a whole to be strongly induced in both 131 children and adults. Children, however, demonstrated stronger upregulation of the B cell and T 132 cell activation pathways, macrophage activation and phagocytosis, and several cytokine 133 production pathways, including IL-2, IL-4, TNF and IFNY. A few immune pathways showed 134 inconsistent expression changes compared to the respective No Virus groups, including 135 neutrophil mediated immunity and mast cell mediated immunity. Intriguingly, both children and 136 adults with SARS-CoV-2 infection exhibited downregulation of olfactory receptor gene expression 137 (sensory perception of chemical stimulus pathway), consistent with the loss of sense of smell 138 clinically observed in both age cohorts $28,29$.

139 We complemented the analyses comparing SARS-CoV-2 and No Virus patients in each 140 cohort separately by directly comparing gene expression between SARS-CoV-2 infected children 141 and adults, controlling for viral load. We identified 5,352 differentially expressed genes at an 142 adjusted p-value $<0.1$ (Supplemental Data 3), and GSEA of the DE results yielded overall similar 143 patterns to those described above (Figure 2B; Supplemental Data 4). In particular, B cell related 144 pathways (B cell activation, humoral immune response), T cell related pathways ( $T$ cell activation, 145 IFN $\gamma$ production, IL-2 and IL-4 production) and chemokine/cytokine signaling were more highly 146 expressed in children with COVID-19.

147 While some immune pathways did not reach statistical significance in the direct 148 comparison between children and adults with SARS-CoV-2, they typically trended in the same 
149 direction (Figure 2B). On the other hand, the stark disparity in neutrophil activation observed in

150 the comparison to the No Virus groups was only weakly supported in the direct comparison, likely

151 reflecting differences among the No Virus patients themselves. The direct comparison clearly

152 revealed lower expression of cilia-associated genes in children with SARS-CoV-2 and also

153 suggested a trend toward lower expression of interferon-stimulated genes, though the pathway

154 just missed the statistical significance cutoff. As expected, many developmental processes

155 unrelated to infection also differed in the direct comparison between children and adults

156 (Supplemental Data 4).

157 Many of the pathways identified in the GSEA results as differentially expressed between

158 children and adults with COVID-19 were tightly related to particular cell types. We therefore 159 applied in silico estimation of cell type proportions ${ }^{30}$ based on marker genes derived from an 160 airway single-cell study ${ }^{31}$ as an additional approach to contextualize our findings (Figure 3, 161 Figure 3-figure supplement 1; Supplemental Data 5).

162 Consistent with the GSEA results, we found that SARS-CoV-2 infection triggered 163 significantly greater recruitment of B cells to the upper airway in children compared to adults, 164 which was also evident in the comparison between children and adults with other respiratory 165 viruses (Figure 3A). In contrast, differences in estimated T cell proportions were much subtler 166 (Figure 3-figure supplement 1), suggesting the GSEA results reflected distinctions in T cell 167 identity and regulation rather than cell number.

168 We previously observed in our adult study that infection with SARS-CoV-2 was associated 169 with blunted recruitment of macrophages and neutrophils to the upper airway as compared to 170 other respiratory viruses ${ }^{22}$, and these trends appear to re-capitulate in children despite the limited 171 sample size of the pediatric Other Virus group (Figure 3B,C). Nevertheless, macrophage 172 proportions trended higher in children with SARS-CoV-2 compared to adults (Figure 3B), as did 173 those of dendritic cells (Figure 3D). 
Intriguingly, while proportions of ciliated cells did not differ between children and adults in

175 the No Virus group, children with SARS-CoV-2 exhibited a marked decrease in ciliated cell 176 proportions that was entirely absent in the adults (Figure 3E), consistent with the GSEA findings.

177 This was accompanied by greater proportions of basal cells in children compared to adults with

178 SARS-CoV-2 (Figure 3F). Recent studies have found that ciliated cells are a major target for 179 SARS-CoV-2 at the onset of infection ${ }^{32,33}$, and a possible interpretation of our results is that 180 children are better able to turn over and clear infected ciliated cells in the nasopharynx, potentially 181 helping them more effectively control the infection.

182 Finally, we examined the effect of SARS-CoV-2 viral load on gene expression in pathways 183 of interest within each age cohort using robust regression. Expression of interferon-stimulated 184 genes (ISGs) frequently correlates with viral load, as we observed in our previous analysis in 185 adults $^{22}$. Children and adults exhibited similar trends for several of the ISGs that most strongly 186 correlated with viral load in our previous analysis, such as CXCL11 and OASL, though the slope trended higher in children (Figure 4A). However, some of the ISGs that were strongly differentially expressed in adults with COVID-19 compared to those with non-viral illnesses exhibited much more gradual activation in the pediatric cohort, such that only children with high viral load achieved comparable expression to that of adults (e.g., IFI6, IFI27; Figure 4B). The "lagging" response of these ISGs in children with lower SARS-CoV-2 viral load explains the trend toward lower pathway expression observed in the GSEA results (Figure 2B). These findings suggest differences in ISG193 specific regulation and/or cellular origins between children and adults that defy simple 194 generalization. While the significance of such subtle distinctions is unclear, they appear less likely 195 to drive the dramatic outcome disparities.

In stark contrast to ISGs, the expression of several B cell marker genes (e.g. CD22, 197 CD79A) was weakly correlated with viral load, especially in children (Figure 4C). These genes 198 exhibited significant heterogeneity between patients, likely reflecting the timing of activation of the 
B cell response, but the fraction of children who were engaging the response at the time of 200 sampling was clearly greater.

Our study has several limitations that should be kept in mind: 1) a larger sample size would have increased the generalizability of our findings; 2) we could not directly control for timing since onset of infection; 3) proteomic measurements may have provided additional insight but were not

204 possible from the available swab specimens, which were collected into a virus inactivating agent;

205 4) our findings regarding tissue cellular composition require validation by single-cell sequencing;

206 and lastly, 5) the majority of subjects with COVID-19 had mild disease at the time of sampling and

207 did not require hospitalization. Results may have differed if specimens from a greater proportion

208 of severely ill individuals had been available, however, this likely resulted in a more relevant 209 comparison since few children develop severe disease.

210 Taken together, our findings suggest children elicit a more robust pro-inflammatory and

211 adaptive immune response to SARS-CoV-2 infection in the upper airway compared to adults but

212 an overall comparable type I interferon response. Children, unlike adults, also display decreased

213 proportions of ciliated cells, the primary targets of SARS-CoV-2, upon infection with the virus.

214 These differences in the upper airway immune response and tissue composition may both 215 contribute to the protection of children from severe disease in the lower respiratory tract. Further 216 study is warranted to unravel why children are better protected specifically against SARS-CoV-2, 217 or perhaps more broadly against $\beta$-coronaviruses ${ }^{34}$, as compared to other common respiratory 218 viruses.

\section{Materials and Methods}

\section{Study design and clinical cohort}

We previously conducted and published an observational cohort study of adult patients 223 with acute respiratory illnesses tested for COVID-19 by RT-PCR at the University of California, 224 San Francisco (UCSF), leveraging leftover RNA extracted from clinical NP swab specimens ${ }^{22}$. 
225 The UCSF Institutional Review Board (IRB) granted a waiver of consent under IRB protocol \#17-

226 24056. For the novel analyses presented here, we leveraged the published adult dataset while 227 supplementing it with pediatric samples similarly obtained at UCSF under the same protocol.

228 Additionally, pediatric samples were obtained from patients tested for COVID-19 by RT-PCR from 229 NP swabs at Children's Hospital Colorado (CHCO). $\mathrm{CHCO}$ specimens and data were obtained 230 under IRB protocols \#0865, \#20-1617 and \#20-0972, which also granted a waiver of consent.

231 Demographic and clinical data for all patients were obtained from a combination of electronic and 232 manual abstraction of medical records at the respective institutions.

233 Children up to 19 years of age and adults at least 40 years of age were eligible for inclusion 234 in the present analysis. Enrollment was not restricted based on sex or race. Some samples were 235 ultimately excluded based on sequencing metrics, as described in the following sections.

\section{Sample processing}

Excess clinical swab specimens were stored in viral transport media at $-80^{\circ} \mathrm{C}$ in the 238 Clinical Microbiology Laboratories. Specimens were thawed and 200uL aliquots of specimen were 239 added to 200uL of DNA/RNA Shield (Zymo Research, Irvine, CA) in sterile $1.5 \mathrm{~mL}$ microtubes 240 with appropriate biohazard precautions.

\section{$241 \quad$ Metagenomic RNA sequencing}

242 Specimens underwent RNA extraction and metagenomic sequencing, as previously 243 described ${ }^{22}$. Briefly, RNA was extracted from $200 \mu \mathrm{L}$ of specimen in DNA/RNA shield using bead244 based lysis and the Zymo Pathogen Magbead kit (Zymo). We also processed negative control 245 samples (water and HeLa cell RNA) to account for background contamination. All samples were 246 spiked with RNA standards from the External RNA Controls Consortium (ERCC) $)^{35}$. Depletion of 247 cytosolic and mitochondrial rRNA was performed using Fastselect (Qiagen, Germantown, MD). 248 RNA was reverse transcribed to generate cDNA and used to construct sequencing libraries using 
249 the NEBNext Ultra II Library Prep Kit (New England Biolabs, Ipswich, MA). Libraries underwent

250146 nucleotide paired-end sequencing on an Illumina Novaseq 6000 instrument.

\section{Metagenomic analysis of respiratory viruses}

Samples were processed through the IDSeq pipeline ${ }^{36,37}$, which performs reference based

253 alignment at both the nucleotide and amino acid level against sequences in the National Center

254 for Biotechnology Information (NCBI) nucleotide (NT) and non-redundant (NR) databases,

255 respectively, followed by assembly of the reads matching each taxon detected. We further

256 processed the results for viruses with established pathogenicity in the respiratory tract ${ }^{38}$. We

257 evaluated whether one of these viruses was present in a patient sample if it met the following

258 three initial criteria: (i) at least 10 counts mapped to NT sequences, (ii) at least 1 count mapped

259 to NR sequences, (iii) average assembly nucleotide alignment length of at least $70 \mathrm{bp}$.

Negative control (water and HeLa cell RNA) samples enabled estimation of the number of

261 background reads expected for each virus, which were normalized by input mass as determined

262 by the ratio of sample reads to spike-in ERCC RNA standards. Viruses were then additionally

263 tested for whether the number of sequencing reads aligned to them in the NT database was

264 significantly greater than background. This was done by modeling the number of background

265 reads as a negative binomial distribution, with mean and dispersion fitted on the negative controls.

266 We estimated the mean parameter of the negative binomial for each taxon (virus) by averaging

267 the read counts across all negative controls after normalizing by ERCCs. We estimated a single

268 dispersion parameter across all taxa using the functions $\mathrm{glm} \cdot \mathrm{nb}()$ and theta.md() from the $\mathrm{R}$

269 package MASS. We considered a sample to have a pathogenic respiratory virus detected by

270 sequencing if the virus achieved an adjusted p-value $<0.05$ after Holm's correction for all tests

271 performed in the same sample. 
We used the IDSeq-calculated viral reads-per-million (rpM), based on the NT alignment,

273 as a uniform measure of SARS-CoV-2 abundance across all samples. A value of $0.1 \mathrm{rpM}$ was

274 added to all samples with $\mathrm{rpM}<0.1$.

\section{Assignment of samples to comparator groups}

276 As in our previous analysis ${ }^{22}$, we divided the samples in each age cohort into three viral

277 status groups: 1) samples with a positive clinical PCR test for SARS-CoV-2 were assigned to the

278 "SARS-CoV-2" group; 2) samples with a negative PCR test for SARS-CoV-2 and no evidence of

279 another pathogenic respiratory virus in the metagenomic sequencing were assigned to the "No

280 Virus" group; and 3) samples with a negative PCR test for SARS-CoV-2 but another respiratory

281 virus detected by sequencing were assigned to the "Other Virus" group.

282 We retained for analysis only samples in the SARS-CoV-2 groups with at least $10 \mathrm{rpM}$,

283 roughly corresponding to PCR $C_{t}$ values below $30^{22}$, to focus on cases with likely active viral

284 replication, where a clear transcriptional response to the virus is expected to be found. This

285 approach was based on the well-established correlation between viral load and recovery of

286 actively replicating virus from respiratory specimens, and more specifically the finding that

287 specimens with a PCR $\mathrm{C}_{\mathrm{t}}<30$ are associated with the ability to culture SARS-CoV-2 $23-25$.

288 Human gene expression quantification

$289 \quad$ Following demultiplexing, sequencing reads were pseudo-aligned with kallisto ${ }^{39}$ (v. 0.46.1;

290 including bias correction) to an index consisting of all transcripts associated with human protein

291 coding genes (ENSEMBL v.99), cytosolic and mitochondrial ribosomal RNA sequences, and the

292 sequences of ERCC RNA standards. Samples were retained for analysis if they had at least

293400,000 estimated counts associated with transcripts of protein coding genes. Gene-level counts

$294(n=19,939)$ were generated from the transcript-level abundance estimates using the R package

295 tximport ${ }^{40}$, with the scaledTPM method. 
Genes were retained for each DE analysis if they had at least 10 counts in at least $20 \%$ 298 of the samples included in the analysis. All analyses were performed with the R package limma ${ }^{41}$, 299 using quantile normalization and the voom method. The design formula for the comparisons within 300 each age cohort was viral status, where viral status was either "SARS-CoV-2" or "No Virus". The 301 design formula for the direct comparison between children and adults with SARS-CoV-2 was $302 \sim \log _{10}(\mathrm{rpM})+$ age cohort, where age cohort was either "children" or "adults".

\section{Gene set enrichment analyses (GSEA)}

304 Gene set enrichment analysis was based on Gene Ontology (GO) biological process 305 pathway annotations ${ }^{27}$, using the non-redundant version available through WebGestalt ${ }^{42}$. Only 306 pathways with a minimum size of 10 genes and a maximum size of 1,500 genes were retained 307 for analysis. The analysis was performed using the fgseaMultilevel function in the R package 308 fgsea ${ }^{43}$, which calculates p-values based on an adaptive, multilevel splitting Monte Carlo scheme.

309 The input consisted of all genes in the respective DE analysis, pre-ranked by fold-change. The 310 gene sets shown in Figure 2 were manually selected to reduce redundancy and highlight diverse

311 biological functions from among those with a Benjamini-Hochberg adjusted $p$-value $<0.05$ in at 312 least one of the analyses. Full results are provided in Supplemental Data 2 and Supplemental 313 Data 4.

314 In silico estimation of cell type proportions

315 Cell-type proportions were estimated using the CIBERSORT $X$ algorithm ${ }^{30}$ based on 316 single cell signatures derived from the human lung cell atlas ${ }^{31}$. Differences in estimated 317 proportions between comparator groups were evaluated for statistical significance using a Mann318 Whitney test with Holm's correction for multiple testing. 
$\underline{\text { Regression of gene counts against viral load }}$

We performed robust regression of the limma-generated quantile normalized gene counts

$321\left(\log _{2}\right.$ scale) against $\log _{10}(\mathrm{rpM})$ of SARS-CoV-2 for selected genes. The analysis was performed

322 within each age cohort separately using the R package robustbase ${ }^{44}$, which implements MM-type 323 estimators for linear regression ${ }^{45,46}$, the KS2014 setting and the model: quantile normalized 324 counts $\left(\log _{2}\right.$ scale $) \sim \log _{10}(\mathrm{rpM})$. Model predictions were generated using the R package ggeffects 325 and used for display in the individual gene plots. Error bands represent normal distribution 95\% 326 confidence intervals around each prediction. Reported p-values for significance of the difference 327 of the regression coefficient from 0 are based on a t-statistic. Reported $R^{2}$ values represent the 328 adjusted robust coefficient of determination ${ }^{47}$.

\section{Data availability}

Gene counts and sample metadata have been deposited under NCBI GEO accession 332 GSE179277. The published human lung single-cell datasets used for cell-type proportions 333 analysis can be obtained through Synapse under accessions syn21560510 and syn21560511.

\section{Funding}

NHLBI K23HL138461-01A1 (CL); Chan Zuckerberg Biohub (AD, AOP, NN, JLD); COVID-

337 Child Health Research Award by the Research Institute at Children's Hospital Colorado; 338 philanthropic support from Mark and Carrie Casey, Julia and Kevin Hartz, Carl Kawaja and Wendy 339 Holcombe, Eric Keisman and Linda Nevin, Martin and Leesa Romo, Diana Wagner, Jerry Yang 340 and Akiko Yamazaki, and Three Sisters Foundation. 
We would like to thank Sam Dominguez, MD, PhD, Kirk Harris, PhD, Aline Maddux, MD,

343 Christina Osborne, MD, and Matthew Leroue, MD, for their input on study design and review of

344 the data.

345

346

\section{References}

347 348

349

350

351

352

353

354

355

356

357

358

359

360

361

362

363

364

365

366

367

368

369

370

371

372

373

374

375

376

377

378

1. O'Driscoll, M. et al. Age-specific mortality and immunity patterns of SARS-CoV-2. Nature 590, 140-145 (2021).

2. Levin, A. T. et al. Assessing the age specificity of infection fatality rates for COVID-19: systematic review, meta-analysis, and public policy implications. Eur J Epidemiol 35, 11231138 (2020).

3. Woolf, S. H., Chapman, D. A. \& Lee, J. H. COVID-19 as the Leading Cause of Death in the United States. JAMA (2020) doi:10.1001/jama.2020.24865.

4. Shi, T. et al. Global, regional, and national disease burden estimates of acute lower respiratory infections due to respiratory syncytial virus in young children in 2015: a systematic review and modelling study. The Lancet 390, 946-958 (2017).

5. Nair, $\mathrm{H}$. et al. Global burden of respiratory infections due to seasonal influenza in young children: a systematic review and meta-analysis. Lancet 378, 1917-1930 (2011).

6. Principi, N. \& Esposito, S. Severe influenza in children: incidence and risk factors. Expert Review of Anti-infective Therapy 14, 961-968 (2016).

7. Matias, G. et al. Estimates of hospitalization attributable to influenza and RSV in the US during 1997-2009, by age and risk status. BMC Public Health 17, 271 (2017).

8. Tin Tin Htar, M., Yerramalla, M. S., Moïsi, J. C. \& Swerdlow, D. L. The burden of respiratory syncytial virus in adults: a systematic review and meta-analysis. Epidemiol Infect 148, e48.

9. Clinical Aspects of Pandemic 2009 Influenza A (H1N1) Virus Infection | NEJM. https://www.nejm.org/doi/10.1056/NEJMra1000449.

10. Ludvigsson, J. F. Systematic review of COVID-19 in children shows milder cases and a better prognosis than adults. Acta Paediatrica 109, 1088-1095 (2020).

11. Bhopal, S. S., Bagaria, J., Olabi, B. \& Bhopal, R. Children and young people remain at low risk of COVID-19 mortality. The Lancet Child \& Adolescent Health 5, e12-e13 (2021).

12. Bailey, L. C. et al. Assessment of 135794 Pediatric Patients Tested for Severe Acute Respiratory Syndrome Coronavirus 2 Across the United States. JAMA Pediatr 175, 176 (2021).

13. $\mathrm{Xu}, \mathrm{Y}$. et al. Characteristics of pediatric SARS-CoV-2 infection and potential evidence for persistent fecal viral shedding. Nat Med 26, 502-505 (2020).

14. Dong, Y. et al. Epidemiology of COVID-19 Among Children in China. Pediatrics 145, (2020).

15. Williamson, E. J. et al. Factors associated with COVID-19-related death using OpenSAFELY. Nature 584, 430-436 (2020). 
16. Weisberg, S. P. et al. Distinct antibody responses to SARS-CoV-2 in children and adults across the COVID-19 clinical spectrum. Nat Immunol 22, 25-31 (2021).

17. Pierce, C. A. et al. Immune responses to SARS-CoV-2 infection in hospitalized pediatric and adult patients. Science Translational Medicine 12, (2020).

18. Madera, S. et al. Nasopharyngeal SARS-CoV-2 viral loads in young children do not differ significantly from those in older children and adults. Sci Rep 11, 3044 (2021).

19. Chung, E. et al. Comparison of Symptoms and RNA Levels in Children and Adults With SARSCoV-2 Infection in the Community Setting. JAMA Pediatrics (2021) doi:10.1001/jamapediatrics.2021.2025.

20. Pierce, C. A. et al. Natural mucosal barriers and COVID-19 in children. JCI Insight 6, (2021).

21. Koch, C. M. et al. Immune response to SARS-CoV-2 in the nasal mucosa in children and adults. $\quad$ http://medrxiv.org/lookup/doi/10.1101/2021.01.26.21250269 doi:10.1101/2021.01.26.21250269.

22. Mick, E. et al. Upper airway gene expression reveals suppressed immune responses to SARS-CoV-2 compared with other respiratory viruses. Nat Commun 11, 5854 (2020).

23. Singanayagam, A. et al. Duration of infectiousness and correlation with RT-PCR cycle threshold values in cases of COVID-19, England, January to May 2020. Eurosurveillance 25, 2001483 (2020).

24. La Scola, B. et al. Viral RNA load as determined by cell culture as a management tool for discharge of SARS-CoV-2 patients from infectious disease wards. Eur J Clin Microbiol Infect Dis 39, 1059-1061 (2020).

25. Yamada, S. et al. Assessment of SARS-CoV-2 infectivity of upper respiratory specimens from COVID-19 patients by virus isolation using VeroE6/TMPRSS2 cells. BMJ Open Respir Res 8, e000830 (2021).

26. Subramanian, A. et al. Gene set enrichment analysis: a knowledge-based approach for interpreting genome-wide expression profiles. Proc Natl Acad Sci U S A 102, 15545-15550 (2005).

27. Ashburner, M. et al. Gene ontology: tool for the unification of biology. The Gene Ontology Consortium. Nat Genet 25, 25-29 (2000).

28. Giacomelli, A. et al. Self-reported Olfactory and Taste Disorders in Patients With Severe Acute Respiratory Coronavirus 2 Infection: A Cross-sectional Study. Clinical Infectious Diseases 71, 889-890 (2020).

29. Kumar, L. et al. Loss of smell and taste in COVID-19 infection in adolescents. Int J Pediatr Otorhinolaryngol 142, 110626 (2021).

30. Newman, A. M. et al. Robust enumeration of cell subsets from tissue expression profiles. Nat Methods 12, 453-457 (2015).

31. Travaglini, K. J. et al. A molecular cell atlas of the human lung from single-cell RNA sequencing. Nature 587, 619-625 (2020).

32. Ravindra, N. G. et al. Single-cell longitudinal analysis of SARS-CoV-2 infection in human airway epithelium identifies target cells, alterations in gene expression, and cell state changes. PLOS Biology 19, e3001143 (2021). 
33. Fiege, J. K. et al. Single cell resolution of SARS-CoV-2 tropism, antiviral responses, and susceptibility to therapies in primary human airway epithelium. PLOS Pathogens 17, e1009292 (2021).

34. Stockman, L. J. et al. Severe acute respiratory syndrome in children. Pediatr Infect Dis J 26, 68-74 (2007).

35. Pine, P. S. et al. Evaluation of the External RNA Controls Consortium (ERCC) reference material using a modified Latin square design. BMC Biotechnology 16, 54 (2016).

36. Ramesh, A. et al. Metagenomic next-generation sequencing of samples from pediatric febrile illness in Tororo, Uganda. PLoS One 14, e0218318 (2019).

37. Kalantar, K. L. et al. IDseq-An open source cloud-based pipeline and analysis service for metagenomic pathogen detection and monitoring. Gigascience 9, (2020).

38. Langelier, C. et al. Integrating host response and unbiased microbe detection for lower respiratory tract infection diagnosis in critically ill adults. Proc Natl Acad Sci U S A 115, E12353-E12362 (2018).

39. Bray, N. L., Pimentel, H., Melsted, P. \& Pachter, L. Near-optimal probabilistic RNA-seq quantification. Nature Biotechnology 34, 525-527 (2016).

40. Soneson, C., Love, M. I. \& Robinson, M. D. Differential analyses for RNA-seq: transcript-level estimates improve gene-level inferences. F1000Res 4, 1521 (2015).

41. Ritchie, M. E. et al. limma powers differential expression analyses for RNA-sequencing and microarray studies. Nucleic Acids Res 43, e47 (2015).

42. Liao, Y., Wang, J., Jaehnig, E. J., Shi, Z. \& Zhang, B. WebGestalt 2019: gene set analysis toolkit with revamped Uls and APIs. Nucleic Acids Research 47, W199-W205 (2019).

43. Korotkevich, G. et al. Fast gene set enrichment analysis. bioRxiv 060012 (2021) doi:10.1101/060012.

44. Maechler, M., Rousseeuw, P., Croux, C., Todorov, V. \& Ruckstuhl, A. robustbase: Basic Robust Statistics. (2021).

45. Koller, M. \& Stahel, W. A. Sharpening Wald-type inference in robust regression for small samples. Computational Statistics \& Data Analysis 55, 2504-2515 (2011).

46. Yohai, V. J. High Breakdown-Point and High Efficiency Robust Estimates for Regression. The Annals of Statistics 15, 642-656 (1987).

47. Renaud, O. \& Victoria-Feser, M.-P. A robust coefficient of determination for regression. Journal of Statistical Planning and Inference 140, 1852-1862 (2010). 
454 Supplemental Table 1A: Adult vs Pediatric cohort characteristics.

\begin{tabular}{|c|c|c|c|c|c|c|}
\hline & $\begin{array}{l}\text { Adults } \\
\text { (All) }\end{array}$ & $\begin{array}{l}\text { Children } \\
\text { (All) }\end{array}$ & $p$ value & $\begin{array}{c}\text { Adults } \\
\text { (SARS-CoV-2) }\end{array}$ & $\begin{array}{c}\text { Children } \\
\text { (SARS-CoV-2) }\end{array}$ & $p$ value \\
\hline Total Enrolled (n) & 154 & 83 & & 45 & 38 & \\
\hline Age, years (median, range, IQR) & $\begin{array}{c}62 \text { (40-89, } \\
47-71)\end{array}$ & $\begin{array}{c}4(<1-19,2- \\
12)\end{array}$ & & $\begin{array}{c}57(40-82,44- \\
64)\end{array}$ & $5(<1-19,2-11)$ & \\
\hline Female gender & $78(51 \%)$ & $42(51 \%)$ & 0.93 & $25(56 \%)$ & $16(42 \%)$ & 0.27 \\
\hline \multicolumn{7}{|l|}{ Clinical Encounter Type } \\
\hline Inpatient & $42(27 \%)$ & $31(37 \%)$ & & $4(9 \%)$ & $1(3 \%)$ & \\
\hline Intensive Care Unit & $16(10 \%)$ & $0(0 \%)$ & & $2(4 \%)$ & $0(0 \%)$ & \\
\hline Emergency Department & $24(16 \%)$ & $19(23 \%)$ & & $3(7 \%)$ & $6(16 \%)$ & \\
\hline Outpatient & $55(36 \%)$ & $31(37 \%)$ & & $24(53 \%)$ & $30(79 \%)$ & \\
\hline Unknown & $17(11 \%)$ & $2(2 \%)$ & 0.59 & $12(27 \%)$ & $1(3 \%)$ & 0.23 \\
\hline \multicolumn{7}{|l|}{ Race } \\
\hline White or Caucasian & $67(44 \%)$ & $34(41 \%)$ & & $6(13 \%)$ & $13(34 \%)$ & \\
\hline Asian & $25(16 \%)$ & $6(7 \%)$ & & $6(13 \%)$ & $1(3 \%)$ & \\
\hline Black or African American & $14(9 \%)$ & $4(5 \%)$ & & $1(2 \%)$ & $2(5 \%)$ & \\
\hline Native Hawaiian or Pacific Islander & $1(1 \%)$ & $2(2 \%)$ & & $1(2 \%)$ & $0(0 \%)$ & \\
\hline American Indian or Alaska Native & $0(0 \%)$ & $1(1 \%)$ & & $0(0 \%)$ & $0(0 \%)$ & \\
\hline Other & $27(18 \%)$ & $29(35 \%)$ & & $17(38 \%)$ & $17(45 \%)$ & \\
\hline Unknown & $20(13 \%)$ & $7(8 \%)$ & 0.013 & $14(31 \%)$ & $5(13 \%)$ & 0.10 \\
\hline \multicolumn{7}{|l|}{ Ethnicity } \\
\hline Not Hispanic or Latino & $109(71 \%)$ & $43(52 \%)$ & & $16(36 \%)$ & $10(26 \%)$ & \\
\hline Hispanic or Latino & $23(15 \%)$ & $34(41 \%)$ & & $14(31 \%)$ & $23(61 \%)$ & \\
\hline Unknown & $22(14 \%)$ & $6(7 \%)$ & $<0.001$ & $15(33 \%)$ & $5(13 \%)$ & 0.06 \\
\hline
\end{tabular}

Values are $n(\%)$ unless otherwise indicated.

458 *Race categories with <1 patient in both groups were excluded from analyses.

459 Unknown values were excluded from analyses. 
Supplemental Table 1B: Adult cohort clinical and demographic characteristics.

\begin{tabular}{|c|c|c|c|c|c|}
\hline & $\begin{array}{l}\text { Cohort } \\
\text { Overall }\end{array}$ & SARS-CoV-2 & Other Virus & No Virus & $p$ value \\
\hline Total Enrolled (n) & 154 & 45 & 28 & 81 & \\
\hline Age, years (median, range, IQR) & $\begin{array}{c}62(40-89 \\
47-71)\end{array}$ & $\begin{array}{c}57(40-82 \\
44-64)\end{array}$ & $\begin{array}{c}61.5(40-89 \\
45-73)\end{array}$ & $\begin{array}{c}62(41-89 \\
52-72)\end{array}$ & 0.75 \\
\hline Female gender & $78(51 \%)$ & $25(56 \%)$ & $10(36 \%)$ & $43(53 \%)$ & 0.21 \\
\hline \multicolumn{6}{|l|}{ Clinical Encounter Type } \\
\hline Inpatient & $42(27 \%)$ & $4(9 \%)$ & $9(32 \%)$ & $29(36 \%)$ & \\
\hline Intensive Care Unit & $16(10 \%)$ & $2(4 \%)$ & $5(18 \%)$ & $9(11 \%)$ & \\
\hline Emergency Department & $24(16 \%)$ & $3(7 \%)$ & $6(21 \%)$ & $15(19 \%)$ & \\
\hline Outpatient & $55(36 \%)$ & $24(53 \%)$ & $8(29 \%)$ & $23(28 \%)$ & \\
\hline Unknown & $17(11 \%)$ & $12(27 \%)$ & $0(0 \%)$ & $5(6 \%)$ & 0.003 \\
\hline \multicolumn{6}{|l|}{ Race } \\
\hline White or Caucasian & $67(44 \%)$ & $6(13 \%)$ & $19(68 \%)$ & $42(52 \%)$ & \\
\hline Asian & $25(16 \%)$ & $6(13 \%)$ & $5(18 \%)$ & $14(17 \%)$ & \\
\hline Black or African American & $14(9 \%)$ & $1(2 \%)$ & $1(4 \%)$ & $12(15 \%)$ & \\
\hline Native Hawaiian or Pacific Islander & $1(1 \%)$ & $1(2 \%)$ & $0(0 \%)$ & $0(0 \%)$ & \\
\hline American Indian or Alaska Native & $0(0 \%)$ & $0(0 \%)$ & $0(0 \%)$ & $0(0 \%)$ & \\
\hline Other & $27(18 \%)$ & $17(38 \%)$ & $3(11 \%)$ & $7(9 \%)$ & \\
\hline Unknown & $20(13 \%)$ & $14(31 \%)$ & $0(0 \%)$ & $6(7 \%)$ & $<0.001^{*}$ \\
\hline \multicolumn{6}{|l|}{ Ethnicity } \\
\hline Not Hispanic or Latino & $109(71 \%)$ & $16(36 \%)$ & $26(93 \%)$ & $67(83 \%)$ & \\
\hline Hispanic or Latino & $23(15 \%)$ & $14(31 \%)$ & $1(4 \%)$ & $8(10 \%)$ & \\
\hline Unknown & $22(14 \%)$ & $15(33 \%)$ & $1(4 \%)$ & $6(7 \%)$ & $<0.001$ \\
\hline
\end{tabular}

Values are $\mathrm{n}(\%)$ unless otherwise indicated.

*patients with Native Hawaiian or Pacific Islander race; and patients with American Indian or Alaska Native race were excluded from

467 Age was analyzed as one-way ANOVA and all categorial variables using chi-squared tests. 


\begin{tabular}{|c|c|c|c|c|c|}
\hline & $\begin{array}{l}\text { Cohort } \\
\text { Overall }\end{array}$ & SARS-CoV-2 & Other Virus & No Virus & $p$ value \\
\hline Total Enrolled (n) & 83 & 38 & 11 & 34 & \\
\hline Age, years (median, range, IQR) & $\begin{array}{c}4(<1-19 \\
2-12)\end{array}$ & $\begin{array}{c}5(<1-19,2- \\
11)\end{array}$ & $\begin{array}{c}3(<1-14,2- \\
11)\end{array}$ & $\begin{array}{c}4(<1-16,2- \\
12)\end{array}$ & 0.97 \\
\hline Female gender & $42(51 \%)$ & $16(42 \%)$ & $7(64 \%)$ & $19(56 \%)$ & 0.38 \\
\hline \multicolumn{6}{|l|}{ Clinical Encounter Type } \\
\hline Inpatient & $31(37 \%)$ & $1(3 \%)$ & $2(18 \%)$ & $28(82 \%)$ & \\
\hline Intensive Care Unit & $0(0 \%$ & $0(0 \%)$ & $0(0 \%)$ & $0(0 \%)$ & \\
\hline Emergency Department & $19(23 \%)$ & $6(16 \%)$ & $8(73 \%)$ & $5(15 \%)$ & \\
\hline Outpatient & $31(37 \%)$ & $30(79 \%)$ & $0(0 \%)$ & $1(3 \%)$ & \\
\hline Unknown & $2(2 \%)$ & $1(3 \%)$ & $1(9 \%)$ & $0(0 \%)$ & $<0.001$ \\
\hline \multicolumn{6}{|l|}{ Race } \\
\hline White or Caucasian & $34(41 \%)$ & $13(34 \%)$ & $4(36 \%)$ & $17(50 \%)$ & \\
\hline Asian & $6(7 \%)$ & $1(3 \%)$ & $1(9 \%)$ & $4(12 \%)$ & \\
\hline Black or African American & $4(5 \%)$ & $2(5 \%)$ & $2(18 \%)$ & $0(0 \%)$ & \\
\hline Native Hawaiian or Pacific Islander & $2(2 \%)$ & $0(0 \%)$ & $1(9 \%)$ & $1(3 \%)$ & \\
\hline American Indian or Alaska Native & $1(1 \%)$ & $0(0 \%)$ & $0(0 \%)$ & $1(3 \%)$ & \\
\hline Other & $29(35 \%)$ & $17(45 \%)$ & $2(18 \%)$ & $10(29 \%)$ & \\
\hline Unknown & $7(8 \%)$ & $5(13 \%)$ & $1(9 \%)$ & $1(3 \%)$ & $0.31^{* *}$ \\
\hline \multicolumn{6}{|l|}{ Ethnicity } \\
\hline Not Hispanic or Latino & $43(52 \%)$ & $10(26 \%)$ & $7(64 \%)$ & $26(76 \%)$ & \\
\hline Hispanic or Latino & $34(41 \%)$ & $23(61 \%)$ & $3(27 \%)$ & $8(24 \%)$ & \\
\hline Unknown & $6(7 \%)$ & $5(7 \%)$ & $1(9 \%)$ & $0(0 \%)$ & $<0.001$ \\
\hline
\end{tabular}




\section{Supplemental Data}

479

480 Supplemental Data 1. Differential expression results between the SARS-CoV-2 and No Virus 481 groups in adults and children separately.

482 Supplemental Data 2. GSEA applied to the differential expression results between the SARS-

483 CoV-2 and No Virus groups in adults and children separately.

484 Supplemental Data 3. Differential expression results directly comparing children and adults with 485 SARS-CoV-2, controlling for viral load.

486 Supplemental Data 4. GSEA applied to the direct comparison between children and adults with 487 SARS-CoV-2.

488 Supplemental Data 5. In silico deconvolution of cell type proportions. 
$491 \quad$ Figure 1: Cohort characteristics

492 A) Number of patients in the SARS-CoV-2, No Virus and Other Virus groups in the adult and 493 pediatric cohorts. B) Age distribution across the three viral status groups in the adult and pediatric 494 cohorts. C) Distribution of SARS-CoV-2 viral load, measured in reads-per-million (rpM), in adult 495 and pediatric SARS-CoV-2 patients. P-value derives from a two-sided Mann-Whitney test. 496 D) Distribution of viruses in the Other Virus groups in the adult and pediatric cohorts. Absolute 497 numbers are provided above each bar while the $y$-axis indicates percentage out of each cohort's 498 Other Virus group. One child had both influenza and rhinovirus and two adults had both RSV and 499 rhinovirus.

500

501 Figure 2: Pathways activated in children and adults upon SARS-CoV-2 infection.

502 A) Normalized enrichment scores of selected GO biological process terms that reached statistical 503 significance (Benjamini-Hochberg adjusted P-value < 0.05) in the GSEA using DE genes between 504 the SARS-CoV-2 and No Virus groups in either the adult or pediatric cohort. Statistical 505 significance is denoted by a black outline around the circle. B) Normalized enrichment scores for 506 the same GO terms as in (A) in the GSEA using DE genes between children and adults with 507 SARS-CoV-2. Dark color bars represent pathways that reached statistical significance 508 (Benjamini-Hochberg adjusted P-value < 0.05). 
Figure 3: Cell-type proportion differences between children and adults.

510 In silico estimation of cell-type proportions in the bulk RNA-sequencing using single-cell

511 signatures. Black lines denote the median. The $y$-axis in each panel was trimmed at the maximum

512 value among all groups of $1.5^{\star} I Q R$ above the third quartile, where IQR is the interquartile range.

513 For each cell type, we formally compared each viral status group between the two age cohorts as

514 well as the No Virus and SARS-CoV-2 groups within each age cohort. Pairwise comparisons were

515 performed with a two-sided Mann-Whitney test followed by Holm's correction for multiple testing.

517 Figure 3-figure supplement 1: Additional cell-types.

518 Additional cell types included in the cell-type proportions analysis.

Figure 4: Relationship of selected immune pathway marker genes to SARS-CoV-2 viral load

521 in children and adults.

522 Scatter plots of normalized gene counts ( $\log _{2}$ scale, y-axis) as a function of SARS-CoV-2 viral

523 load $\left(\log _{10}(\mathrm{rpM}), x\right.$-axis) in each age cohort. The viral status group is indicated by the dot color.

524 Robust regression was performed on SARS-CoV-2 patients to characterize the relationship to 525 viral load. Shaded bands represent 95\% confidence intervals. Numerical results listed for each 526 gene refer to, from top to bottom: the regression slope, the $p$-value for the difference of the slope 527 from 0 , and the adjusted robust coefficient of determination $\left(R^{2}\right)$. A) Canonical type I interferon 528 response genes showing high correlation to viral load in adults and children. B) Interferon529 stimulated genes showing a more gradual response to viral load in children compared to adults. 530 C) B cell marker genes. 


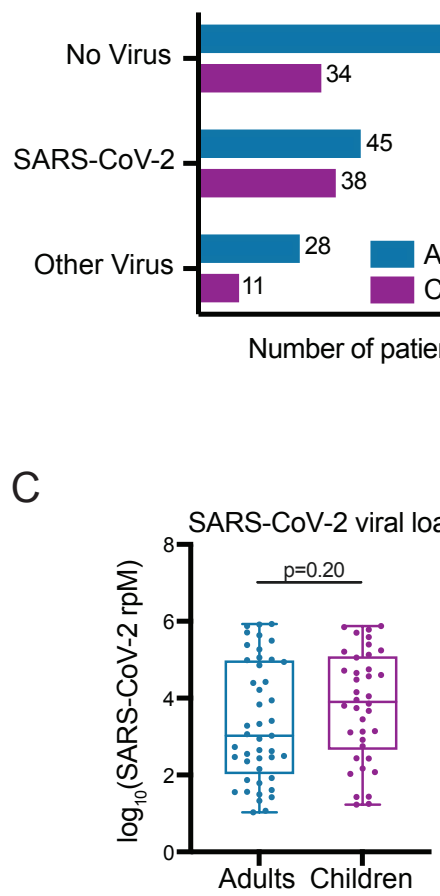

81

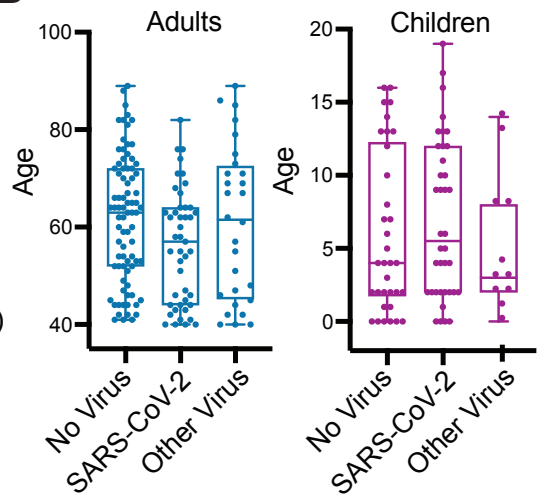

\section{D}

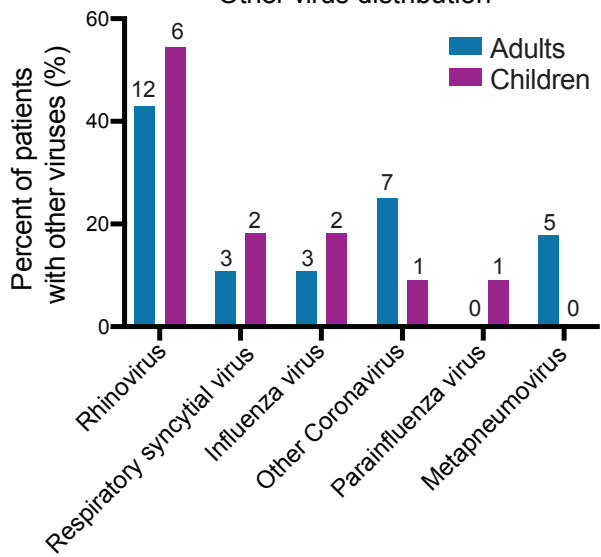

Figure 1 


\section{SARS-CoV-2+ Adults SARS-CoV-2+ Children}

mast cell mediated immunity

neutrophil mediated immunity cellular response to vascular endothelial growth factor stimulus

NIK/NF-kappaB signaling regulation of epithelial cell differentiation phagocytosis response to interleukin-7

$B$ cell activation

inflammatory response to antigenic stimulus cytokine metabolic process interleukin-4 production lymphocyte activation involved in immune response regulation of leukocyte activation regulation of cell-cell adhesion

$\mathrm{T}$ cell activation interleukin-1 production macrophage activation response to tumor necrosis factor interferon-gamma production interleukin-2 production interleukin-10 production myeloid cell differentiation adaptive immune response interleukin-6 production natural killer cell activation cell chemotaxis cytokine secretion

interleukin-12 production regulation of inflammatory response regulation of response to cytokine stimulus myeloid dendritic cell activation regulation of immune effector process regulation of innate immune response humoral immune response cell killing response to chemokine response to interferon-gamma response to type I interferon antigen processing and presentation sensory perception of chemical stimulus

cilium organization
complex assembly mitochondrial respiratory chain complex assembly

Figure 2

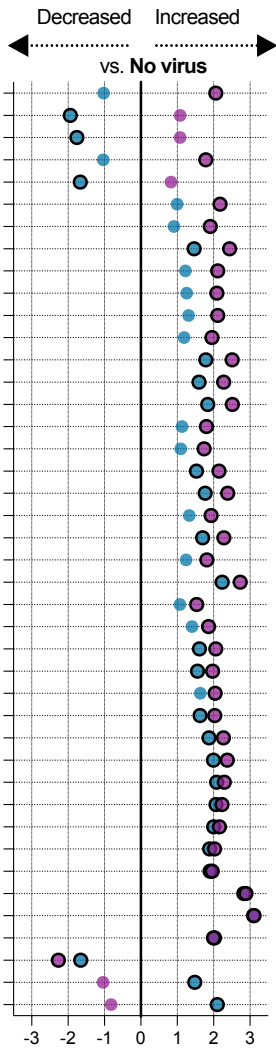

GSEA normalized enrichment score
Higher in adults Higher in children

Among SARS-CoV-2+

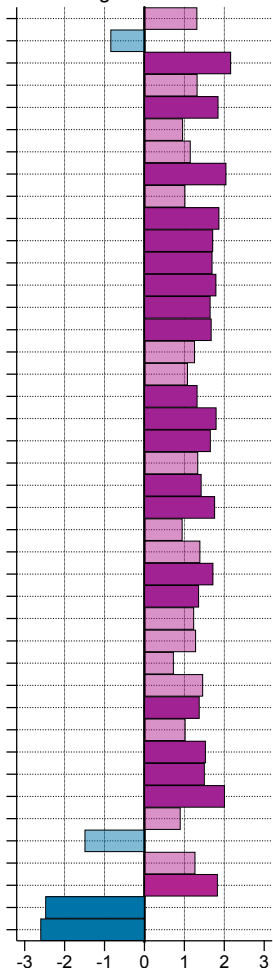

GSEA normalized enrichment score 
A

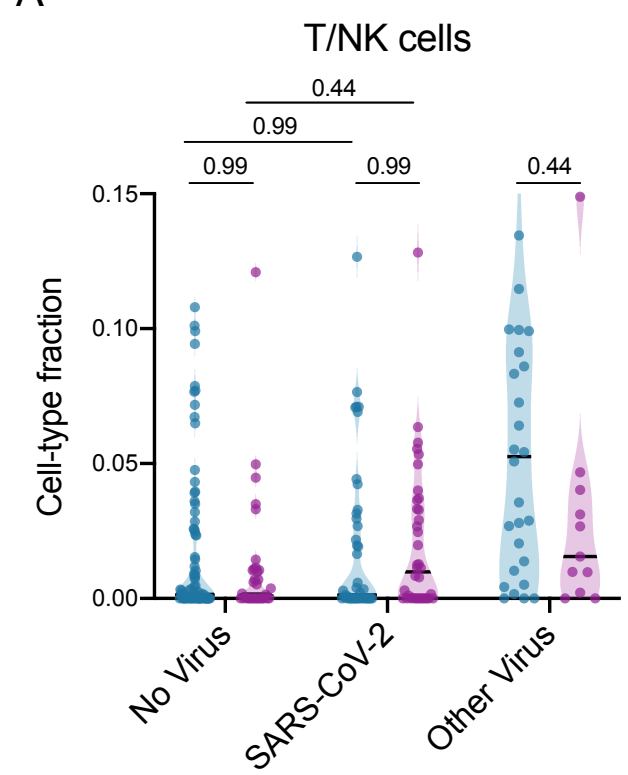

D

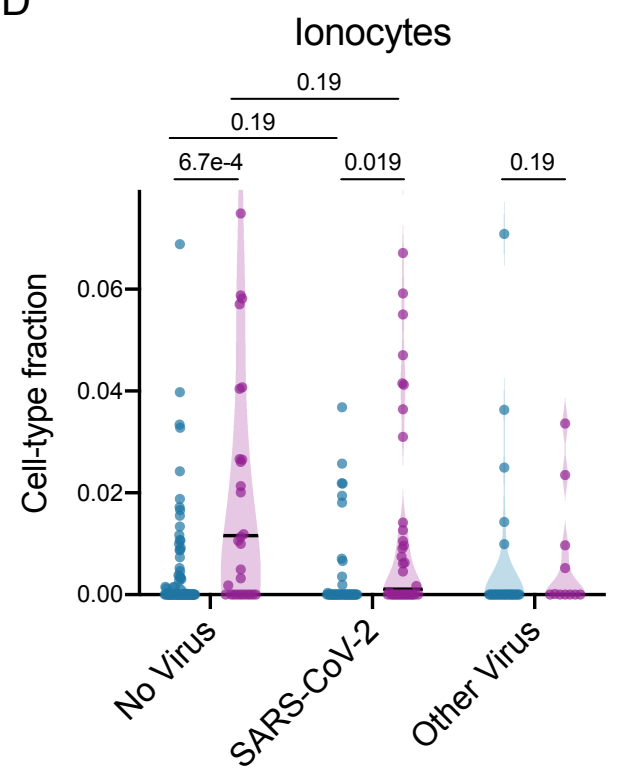

B

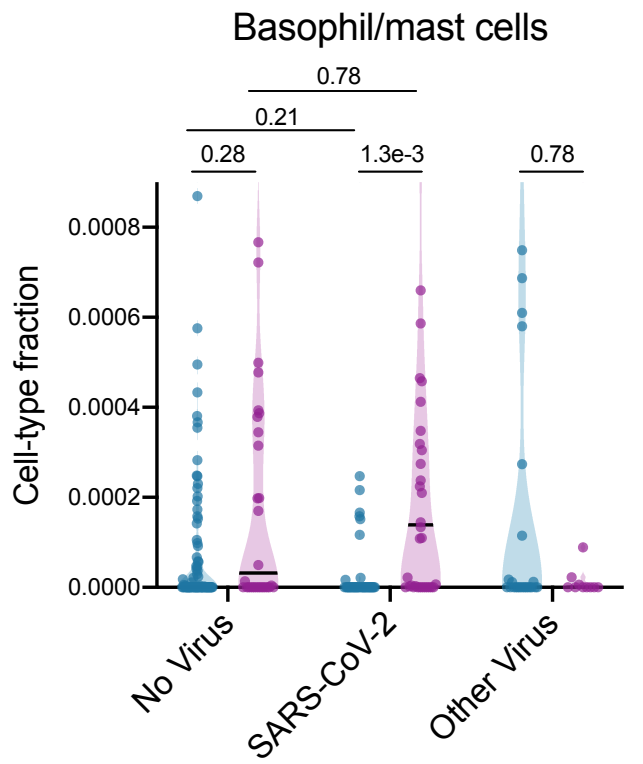

E

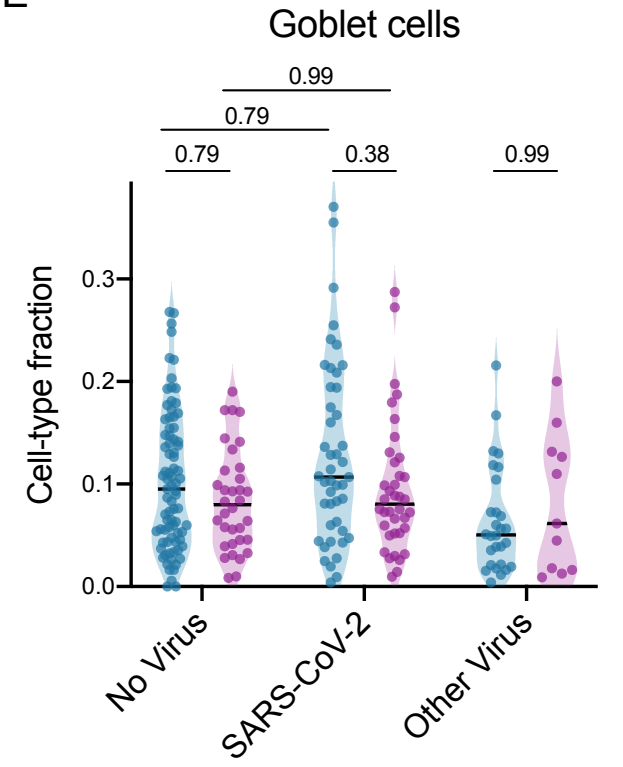

C

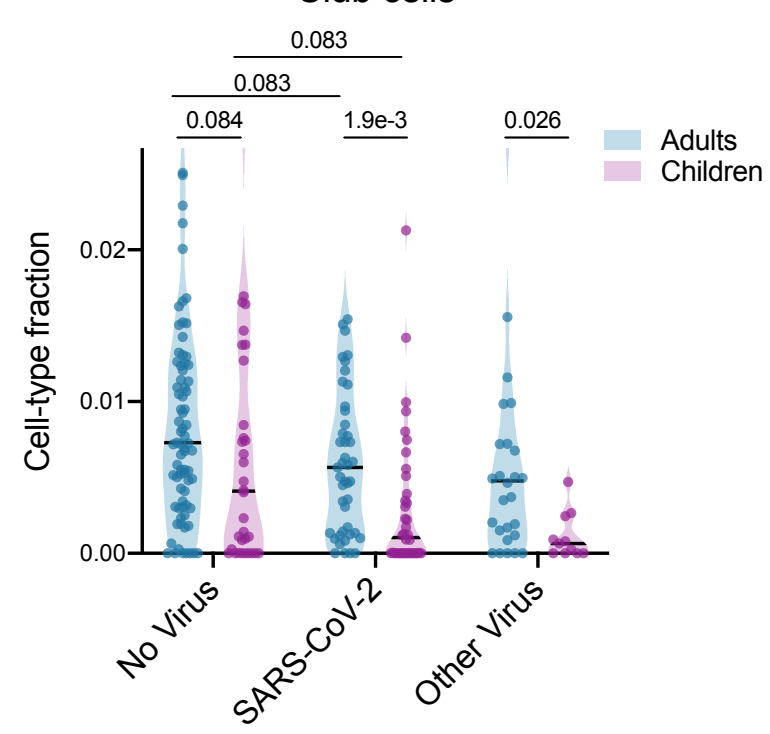


Adults

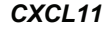

Children
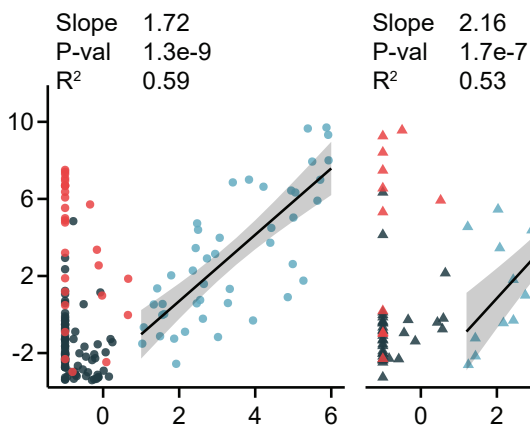

$\begin{array}{ll}\mathrm{R}^{2} & 0.53\end{array}$

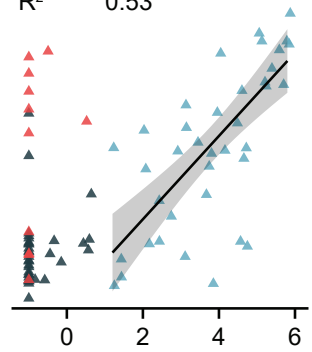

SARS-CoV-2 $\log _{10}(\mathrm{rpM})$
IFI6

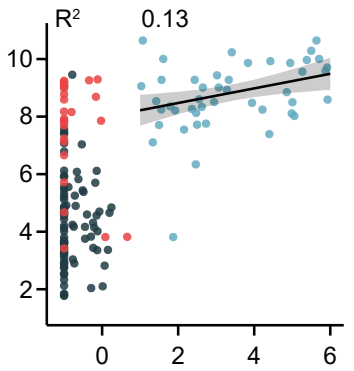

Adults

\section{Slope 0.25}

P-val 9e-3

SARS-CoV-2 $\log _{10}(\mathrm{rpM})$

Children

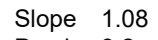

P-val $3.2 \mathrm{e}-11$

$\begin{array}{ll}\mathrm{R}^{2} & 0.7\end{array}$

CD22

Adults

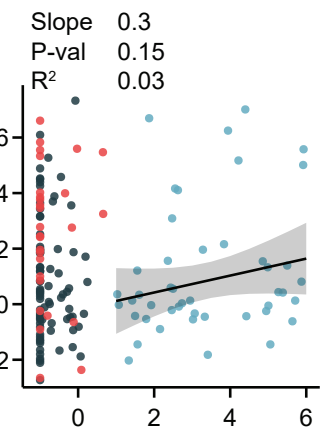

Slope -0.02

P-val 0.96

$\mathrm{R}^{2} \quad 0$

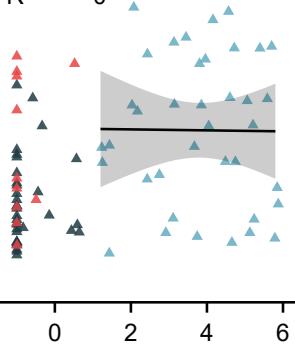

SARS-CoV-2 $\log _{10}(\mathrm{rpM})$

Adults

OASL

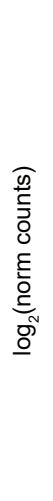

IFI27

Children

Slope 1.16

P-val $6.9 e-6$

$\begin{array}{ll}\mathrm{R}^{2} & 0.42\end{array}$

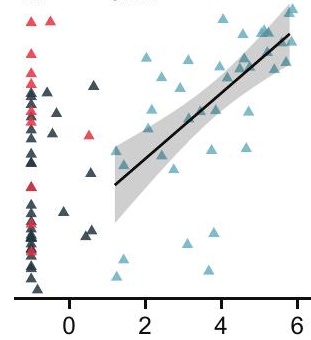

SARS-CoV-2 $\log _{10}(\mathrm{rpM})$
Adults

\section{Slope 0.1 \\ P-val 0.32}

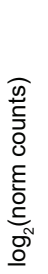

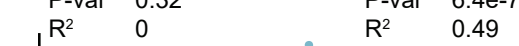

$\begin{array}{ll}R^{2} & 0.49\end{array}$
Children

Slope 0.63

P-val $\quad 6.4 \mathrm{e}-7$

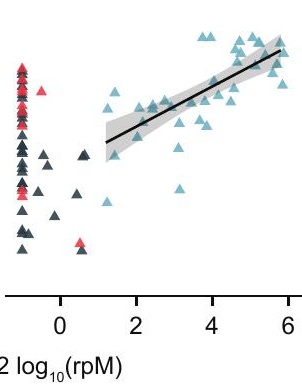

CD79A
Adults

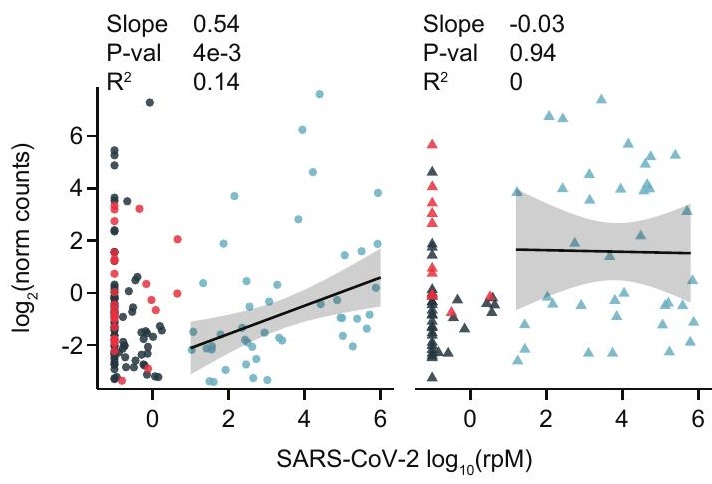

- No virus

- SARS-CoV-2

- Other virus 


\section{Supplementary Files}

This is a list of supplementary files associated with this preprint. Click to download.

- SupplementaryData1covidvsnovirusDE.xlsx

- SupplementaryData2covidvsnovirusfgsea.xlsx

- SupplementaryData3covidpediatricvsadultDE.xlsx

- SupplementaryData4covidpediatricvsadultfgsea.xlsx

- SupplementaryData5deconvolutionresults.xlsx 